\title{
Clinical characteristics and prognostic value of MEX3A mRNA in liver cancer
}

\author{
Dingquan Yang ${ }^{1}$, Yan Jiao ${ }^{\text {Corresp., } 2}$, Yanqing $\mathbf{L i}^{3}$, Xuedong Fang ${ }^{\text {Corresp. } 1}$ \\ ${ }^{1}$ Department of Gastrointestinal Colorectal and Anal Surgery, China-Japan Union Hospital of Jilin University, Changchun, Jilin, China \\ 2 Department of Hepatobiliary and Pancreatic Surgery, The First Hospital of Jilin University, Changchun, Jilin, China \\ 3 Department of Pathophysiology, College of Basic Medical Sciences, Jilin University, Changchun, Jilin, China \\ Corresponding Authors: Yan Jiao, Xuedong Fang \\ Email address: jiaoyan16@mails.jlu.edu.cn, fangxd@jlu.edu.cn
}

Background. MEX3A is an RNA-binding proteins (RBPs) that promotes the proliferation, invasion, migration and viability of cancer cells. The aim of this study was to explore the clinicopathological characteristics and prognostic significance of MEX3A mRNA expression in liver cancer. Methods. RNA-Seq and clinical data were collected from The Cancer Genome Atlas (TCGA). Boxplots were used to represent discrete variables of MEX3A. Chisquare tests were used to analyze the correlation between clinical features and MEX3A expression. Receiver operating characteristic (ROC) curves were used to confirm diagnostic ability. Independent prognostic ability and values were assessed using Kaplan-Meier curves and Cox analysis. Results. We acquired MEX3A RNA-Seq from 50 normal liver tissues and 373 liver cancer patients along with clinical data. We found that MEX3A was up-regulated in liver cancer which increased according to histological grade $(p<0.001)$. MEX3A showed moderate diagnostic ability for liver cancer ( $A U C=0.837)$. Kaplan-Meier curves and Cox analysis revealed that the high expression of MEX3A was significantly associated with poor survival (OS and RFS) $(p<0.001)$. Moreover, MEX3A was identified as an independent prognostic factor of liver cancer $(p<0.001)$. Conclusions. MEX3A expression shows promise as an independent predictor of liver cancer prognosis. 
1 Clinical characteristics and prognostic value of MEX3A mRNA in liver cancer

2 Dingquan Yang ${ }^{1}$, Yan Jiao ${ }^{2,}$, , Yanqing $\mathrm{Li}^{3}$, Ying $\mathrm{Liu}^{4}$ and Xuedong Fang ${ }^{1, *}$

3

4

1 Department of Gastrointestinal Colorectal and Anal Surgery, China-Japan Union Hospital of Jilin University, Changchun, Jilin 130033, China

2 Department of Hepatobiliary and Pancreatic Surgery, The First Hospital of Jilin University, Changchun, Jilin 130021, China

3 Department of Pathophysiology, College of Basic Medical Sciences, Jilin University, Changchun, Jilin 130021, China

4 Department of General Surgery, The Affiliated Hospital to Changchun University of Chinese Medicine, Changchun, Jilin 130021, China

* Correspondence to Xuedong Fang, MD, Ph.D., E-mail address: fangxd@jlu.edu.cn; Mail address: No. 126 XianTai street, Changchun, Jilin, China; Telephone: +86-431-8879-6553, Department of Gastrointestinal Colorectal and Anal Surgery, China-Japan Union Hospital of Jilin University, Changchun, Jilin 130033, China.

And Yan Jiao, Ph.D., E-mail address: lagelangri1@126.com; Mail address: No. 71 XinMin street, Changchun, Jilin, China; Telephone: +86-431-85612345, Department of Hepatobiliary and Pancreatic Surgery, The First Hospital of Jilin University, Changchun, Jilin 130021, China. 
Abstract:

Background. MEX3A is an RNA-binding proteins (RBPs) that promotes the proliferation, invasion, migration and viability of cancer cells. The aim of this study was to explore the clinicopathological characteristics and prognostic significance of MEX3A mRNA expression in liver cancer.

Methods. RNA-Seq and clinical data were collected from The Cancer Genome Atlas (TCGA). Boxplots were used to represent discrete variables of MEX3A. Chi-square tests were used to analyze the correlation between clinical features and MEX3A expression. Receiver operating characteristic (ROC) curves were used to confirm diagnostic ability. Independent prognostic ability and values were assessed using Kaplan-Meier curves and Cox analysis.

Results. We acquired MEX3A RNA-Seq from 50 normal liver tissues and 373 liver cancer patients along with clinical data. We found that MEX3A was up-regulated in liver cancer which increased according to histological grade $(\mathrm{p}<0.001)$. MEX3A showed moderate diagnostic ability for liver cancer $(\mathrm{AUC}=0.837)$. Kaplan-Meier curves and Cox analysis revealed that the high expression of MEX3A was significantly associated with poor survival (OS and RFS) $(p<0.001)$. Moreover, MEX3A was identified as an independent prognostic factor of liver cancer $(p<0.001)$.

Conclusions. MEX3A expression shows promise as an independent predictor of liver cancer prognosis.

\section{Keywords MEX3A, liver cancer, prognosis, RNA-binding proteins, The Cancer Genome} Atlas

\section{Introduction}

Liver cancer is a malignant cancer with poor prognosis that is responsible for more than 780,000 deaths annually, making it the second most common cause of cancer-related mortality worldwide (Bray et al. 2018). Although liver cancer can be alleviated or cured through hepatectomy, orthotopic liver transplantation, and/or ablative procedures (Hanouneh et al. 2019). More than $65 \%$ of patients fail to be cured and frequent recurrence contributes to poor survival. Predicting the overall 1-year survival rates for liver cancer have remained a challenge (Galle PR et al. 2018; Hanouneh et al. 2019). New biomarkers that can predict liver cancer recurrence are urgently required to improve prognosis.

Liver cancer is influenced by post-transcriptional mechanisms that dynamically regulate protein expression. (Wong et al. 2018; Goldstrohm et al. 2018) Cis-regulatory RNA elements and trans-acting factors (Gerstberger et al. 2014; Moore 2005) including RNA-binding proteins (RBPs) play an essential role in gene expression in cancer cells (Masuda \& Kuwano 2019). Recently, a group of RBPS termed MEX-3 RNA binding family member (MEX3) was identified in the nematode Caenorhabditis elegans (Ciosk et al. 2006) and revealed one of the few RBPs with carcinogenic or tumor suppressor activity (Kim et al. 2009; Pereira et al. 2013a).

MEX3 proteins are evolutionarily conserved RNA-binding proteins that consist of four homologous genes (MEX3A-D) (Buchet-Poyau et al. 2007; Courchet et al. 2008; Pereira et al. 2013a). They contained highly conservative one carboxy-terminal RING finger module and two K homology domains, the former mediating E3 ubiquitin ligase activity, the latter providing RNAbinding capacity (Buchet-Poyau et al. 2007). Available evidence implicates the MEX3 family in 
epithelial homeostasis, embryonic development, metabolism, immune responses and cancer, but the specific mechanisms of these effects require elucidation (Pereira et al. 2013a). MEX3A is a member of the MEX3 family (also known as RKHD4 or RNF162) that is expressed in endometrium tissue and the ovaries. MEX3A is a novel component of GW-182 or Dcp-containing bodies in mammals that represent cellular sites of mRNA degradation and the sequestration of non-translated transcripts (Cougot et al. 2004; Eystathioy et al. 2002; Sheth \& Parker 2003).

MEX3A mRNA was recently shown to be overexpressed in Wilms tumors (Krepischi et al. 2016), gastric cancer (Jiang et al. 2012), bladder cancer (Huang et al. 2017), and bladder urothelial carcinoma (Shi \& Huang 2017). MEX3A promotes cell proliferation in bladder (Huang et al. 2017) and gastric (Jiang et al. 2012) cancer and shows potential as a biomarker to predict carcinogenesis (Pereira et al. 2013a). In this study, we analyzed the expression of MEX3A in liver cancer and assessed its clinicopathological potential. We further investigated the potential of MEX3A as an independent predictor of liver cancer prognosis.

\section{Methods}

\section{Clinical and RNA-Seq analysis}

We downloaded the all RNA-Seq expression matrix from the Cancer Genome Atlas (TCGA) database and obtained MEX3A mRNA expression data from liver cancer vs. normal liver tissue using the matrix. We further obtained corresponding clinical and pathological information from TCGA database. The basic clinical data included age, gender, histological grade, stage, T/N/M classification and vital status. MEX3A mRNA expression were estimated as $\log 2(\mathrm{x}+1)$ values and transformed RSEM normalized counts.

\section{Statistical analyses}

We retrospectively analyzed all data using $\mathrm{R}$ (version 3.5.1) (Team 2009). We used nonparametric rank sum tests to analyze MEX3A mRNA expression levels according to different variables and boxplots were visualized. Wilcoxon rank sum tests were used to compare the two subgroups, including disease, age, gender and vital status. Kruskal-Wallis tests were used for the comparison of three or more subgroups, including clinical stage, histologic grade and T/N/M classification. We used the pROC package to draw ROC curves for the evaluation of MEX3A diagnosis through the calculation of AUC values and the measurement of optimal cutoff point to divide samples into high and low MEX3A expression groups (Robin et al. 2011). Further, correlation between clinical features and MEX3A expression group were analyzed through chisquare tests with Fisher's exact test.

To evaluate prognosis, Kaplan-Meier curves were used based on log-rank tests to compare differences in survival status, including overall survival (OS) and relapse-free survival (RFS) between the high and low MEX3A expression groups using the survival package in R (Therneau \& April 1994; Therneau \& Grambsch 2000). Univariate Cox analysis was used to select factors associated with prognosis, with calculations of hazard ratios (HRs) and 95\% confidence intervals (95\% CIs). Independent prognostic values of OS and RFS in the patients were determined through Multivariate Cox analysis. P-values $<0.05$ were deemed statistically significant.

\section{Results}

\section{Clinical characteristics and RNA-Seq analysis}


A total of 423 tissue samples with MEX3A mRNA expression data, including 373 liver cancer and 50 normal liver tissues were obtained from the TCGA. All patients were diagnosed with primary liver cancer. Corresponding patient demographic and clinical characteristics such as age, gender, histologic grade, TNM stage, vital status, and radiation therapy were obtained. All patient data is shown in Table 1.

\section{MEX3A is highly expressed in liver cancer.}

In liver cancer, MEX3A mRNA expression level was significantly up-regulated compared to normal tissues $(p=1.1 \mathrm{e}-14$; Figure $1 \mathrm{~A})$ and increased with higher histological grades $(\mathrm{p}=0.00016$; Figure 1C). MEX3A expression level was significantly associated with vital status $(\mathrm{p}=0.032$; Figure 1I) and age ( $p=0.0011$; Figure $1 G)$.

\section{MEX3A as a liver cancer diagnostic.}

ROC curve analysis showed that MEX3A had moderate diagnostic ability in patients with liver cancer $(\mathrm{AUC}=0.837$; Figure $2 \mathrm{~A})$. The diagnostic ability of MEX3A was comparable in all clinical stages (stage I: $\mathrm{AUC}=0.823$, stage II: $\mathrm{AUC}=0.844$, stage III: $\mathrm{AUC}=0.835$, stage $\mathrm{IV}$ : AUC $=0.888$; Figure 2 B-E).

\section{Relationship between MEX3A expression and clinical characteristics}

Figure S1 showed that the cutoff point was 7.266 when samples were divided according to high and low MEX3A expression. Chi-square tests were used to analyze clinical variables between the two groups, in which high MEX3A expression was associated with cancer related mortality $(p=0.001$; Table 2). High MEX3A expression was also associated with a deterioration in liver tumor histopathology ( $<<0.001$; Table 2$)$.

\section{MEX3A is an independent prognostic to evaluate the survival of liver cancer patients.}

Kaplan-Meier curves showed that patients with high MEX3A expression were more likely to have a poor OS $(p<0.0001$; Figure $3 \mathrm{~A})$. Further subgroup analysis showed that high MEX3A expression was associated with poor OS for all variables: stage I/ II ( $p=0.0011$; Figure $3 \mathrm{~B}$ ), stage III / IV ( $p=0.00022$; Figure 3C), stage G1/G2 ( $<<0.0001$; Figure 3D), stage G3/G4 ( $p=0.044$; Figure $3 E)$, male $(p<0.0001$; Figure $3 F)$, female $(p=0.0066$; Figure $3 G)$, younger $(p=0.0026$; Figure $3 \mathrm{H})$, older $(\mathrm{p}=0.00022$; Figure $3 \mathrm{I})$. Through Univariate and Multivariate Cox analysis of OS, MEX3A $(\mathrm{HR}=2.26,95 \%$ CI [1.58-3.23], $\mathrm{p}<0.0001)$ was identified as an independent risk factor for the prognosis of liver cancer along with $\mathrm{T}$ stage $(\mathrm{p}<0.0001)$ and residual tumors $(p=0.026$; Table 3).

Based on the OS, we further explored the connection between RFS and MEX3A expression, and found that high MEX3A expression was associated with poor RFS ( $<<0.0001$; Figure 4A). RFS was related to the expression of MEX3A for some variables, including stage I/ II ( $p=0.013$; Figure 4B), stage III / IV ( $\mathrm{p}=0.00027$; Figure 4C), stage G1/G2 ( $<0.0001$; Figure 4D), male $(p=0.0023$; Figure $4 F)$, female $(p=0.0049$; Figure $4 G)$, younger $(p=0.0025$; Figure $4 H)$ and older $(\mathrm{p}=0.0047$; Figure 4I). Univariate and Multivariate Cox analysis suggested that MEX3A $(\mathrm{HR}=$ $2.19,95 \%$ CI [1.54-3.12], $\mathrm{p}<0.0001)$ was an independent risk factor to evaluate the RFS for liver cancer along with $\mathrm{T}$ stage $(\mathrm{p}<0.0001)$ and residual tumor status $(\mathrm{p}=0.024$; Table 4$)$.

\section{Discussion}

Liver cancer is one of the deadliest tumors worldwide. The main risk factors for liver cancer 
158 include hepatitis $\mathrm{B}$ or $\mathrm{C}$ virus infections, the intake of aflatoxin, alcohol abuse and non-alcoholic 159 fatty liver disease (NAFLD) (Zhang et al. 2019). Due to the lack of effective diagnostic and 160 prognostic evaluation methods, the mortality rates of liver cancer patients have gradually 161 increased. New molecular markers that can guide prognosis and improve the survival rates of liver 162 cancer patients are urgently required. Our team has devoted to exploring diagnostic and prognostic 163 biomarkers in various cancers (Hou et al. 2019; Jiao et al. 2018; Jiao et al. 2019a; Jiao et al. 2019b; 164 Jiao et al. 2019c; Jiao et al. 2019d; Li et al. 2019; Sun et al. 2019). In this study, MEX3A mRNA 165 was identified as overexpressed in liver cancer tissue and could effectively evaluate the prognosis 166 of liver cancer patients as an independent predictor. A strong correlation between high MEX3A

MEX3A is known to be upregulated in Wilms renal cancer (Krepischi et al. 2016), gastric cancer (Jiang et al. 2012), bladder cancer (Huang et al. 2017) and bladder urothelial cancer (Shi \& Huang 2017). These results are consistent with our finding that MEX3A mRNA is overexpressed in liver cancer $\left(\mathrm{p}=1.1 \mathrm{e}^{-14}\right)$. Area under the ROC curves was 0.837 , which provided evidence that MEX3A was a potential biomarker for liver cancer diagnosis. Interestingly, the expression of MEX3A was higher with increased histological grade $(p<0.0001)$, suggesting that MEX3A is related to tumor progression.

The molecular mechanism underlying the oncogenic effects of MEX3A remain poorly understood. Huang et al. found that MEX3A silencing significantly inhibits the proliferation of bladder cancer cells and promotes apoptosis (Huang et al. 2017). Jiang et al. similarly reported that MEX3A silencing delays the cell cycle progression of gastric cancer cells. MEX3A silencing significantly inhibited cell migration and anchorage-independent growth (Jiang et al. 2012). In colorectal cells, MEX3A is a stemness-related gene (Barriga et al. 2017; Chatterji \& Rustgi 2018; Fernandez-Barral et al. 2019) that acts as a repressive factor through controlling the expression of CDX2. CDX2 inhibits colorectal tumor cells growth, invasion, progression and migration and plays an essential regulatory role in intestinal homeostasis (Bonhomme et al. 2003; Brabletz et al. 2004; Gross et al. 2008; Pereira et al. 2013b). If MEX3A is overexpressed in colonic cell lines, cell polarity and differentiation become impaired leading to carcinogenesis (Pereira et al. 2013b). Combined with our findings, the role of MEX3A in cancer progression explains its clinical links to poor histological grade and poor patient prognosis in liver cancer. It is therefore necessary to explore the relationship between MEX3A and survival.

The MEX3 family shows promise as a biomarker for both cancer progression and prognosis (Pereira et al. 2013a). However, in studies by Huang et al. (Shi \& Huang 2017), no prognostic significance for cancer bladder urothelial carcinoma samples following MEX3A overexpression were observed. These results contrasted our findings and may highlight differential roles of MEX3A in cancer progression according to cancer-type. Of note, upon assessment of each subvariable group, Kaplan-Meier curves revealed that the liver cancer patients with high MEX3A expression had a poor OS. RFS were evaluated according to MEX3A expression and showed a similar relationship with OS, excluding G3/G4 group. This highlights the unique superiority of MEX3A expression for the assessment of liver cancer survival. In particular, as shown in Table 3 and 4, MEX3A may represent a prognostic marker for liver cancer survival (OS and RFS) under 
the strong confounding effects of clinicopathological features, providing useful references for clinicians to aid the development of individualized patient's treatments.

In summary, this is the first study to report the association between MEX3A mRNA and the clinical characteristics and survival of liver cancer patients. MEX3A has great potential to predict the prognosis of liver cancer patients. Future studies should explore the mechanisms by which MEX3A promotes liver cancer in vivo and in vitro. Further clinicopathological information and corresponding clinical tissue samples should be obtained to further validate these findings and to establish MEX3A as a novel prognostic for patients with liver cancer.

\section{Conclusions}

This is the first study to investigate the expression of MEX3A mRNA in liver cancer, revealing its association with specific clinical features. Moreover, our results indicate that MEX3A plays a significant role in the prognosis of liver cancer and can be used as an independent factor to predict liver cancer progression.

\section{Acknowledgements}

\section{No}

\section{References:}

Barriga FM, Montagni E, Mana M, Mendez-Lago M, Hernando-Momblona X, Sevillano M, Guillaumet-Adkins A, Rodriguez-Esteban G, Buczacki SJA, Gut M, Heyn H, Winton DJ, Yilmaz OH, Attolini CS, Gut I, and Batlle E. 2017. Mex3a Marks a Slowly Dividing Subpopulation of Lgr5+ Intestinal Stem Cells. Cell Stem Cell 20:801816.e807. 10.1016/j.stem.2017.02.007

Bonhomme C, Duluc I, Martin E, Chawengsaksophak K, Chenard MP, Kedinger M, Beck F, Freund JN, and DomonDell C. 2003. The $\mathrm{Cdx2}$ homeobox gene has a tumour suppressor function in the distal colon in addition to a homeotic role during gut development. Gut 52:1465-1471.

Brabletz T, Spaderna S, Kolb J, Hlubek F, Faller G, Bruns CJ, Jung A, Nentwich J, Duluc I, Domon-Dell C, Kirchner T, and Freund JN. 2004. Down-regulation of the homeodomain factor Cdx2 in colorectal cancer by collagen type I: an active role for the tumor environment in malignant tumor progression. Cancer Res 64:6973-6977. 10.1158/0008-5472.CAN-04-1132

Bray F, Ferlay J, Soerjomataram I, Siegel RL, Torre LA, and Jemal A. 2018. Global cancer statistics 2018: GLOBOCAN estimates of incidence and mortality worldwide for 36 cancers in 185 countries. CA Cancer J Clin 68:394424. 10.3322/caac.21492

Buchet-Poyau K, Courchet J, Le Hir H, Seraphin B, Scoazec JY, Duret L, Domon-Dell C, Freund JN, and Billaud M. 2007. Identification and characterization of human Mex-3 proteins, a novel family of evolutionarily conserved RNA-binding proteins differentially localized to processing bodies. Nucleic Acids Res 35:1289-1300. 10.1093/nar/gkm016

Chatterji P, and Rustgi AK. 2018. RNA Binding Proteins in Intestinal Epithelial Biology and Colorectal Cancer. Trends Mol Med 24:490-506. 10.1016/j.molmed.2018.03.008

Ciosk R, DePalma M, and Priess JR. 2006. Translational regulators maintain totipotency in the Caenorhabditis elegans germline. Science 311:851-853. 10.1126/science.1122491 
Cougot N, Babajko S, and Seraphin B. 2004. Cytoplasmic foci are sites of mRNA decay in human cells. J Cell Biol 165:31-40. 10.1083/jcb.200309008

Courchet J, Buchet-Poyau K, Potemski A, Bres A, Jariel-Encontre I, and Billaud M. 2008. Interaction with 14-3-3 adaptors regulates the sorting of hMex-3B RNA-binding protein to distinct classes of RNA granules. J Biol Chem 283:32131-32142. 10.1074/jbc.M802927200

Eystathioy T, Chan EK, Tenenbaum SA, Keene JD, Griffith K, and Fritzler MJ. 2002. A phosphorylated cytoplasmic autoantigen, GW182, associates with a unique population of human mRNAs within novel cytoplasmic speckles. Mol Biol Cell 13:1338-1351. 10.1091/mbc.01-11-0544

Fernandez-Barral A, Costales-Carrera A, Buira SP, Jung P, Ferrer-Mayorga G, Larriba MJ, Bustamante-Madrid P, Dominguez O, Real FX, Guerra-Pastrian L, Lafarga M, Garcia-Olmo D, Cantero R, Del Peso L, Batlle E, Rojo F, Munoz A, and Barbachano A. 2019. Vitamin D differentially regulates colon stem cells in patient-derived normal and tumor organoids. FEBS J. 10.1111/febs.14998

Galle PR, Forner A, Llovet JM, Mazzaferro V, Piscaglia F, Raoul JL, Schirmacher P, and V. V. 2018. EASL Clinical Practice Guidelines: Management of hepatocellular carcinoma. J Hepatol 69:182-236. 10.1016/j.jhep.2018.03.019

Gerstberger S, Hafner M, and Tuschl T. 2014. A census of human RNA-binding proteins. Nat Rev Genet 15:829-845. $10.1038 / \mathrm{nrg} 3813$

Goldstrohm AC, Hall TMT, and McKenney KM. 2018. Post-transcriptional Regulatory Functions of Mammalian Pumilio Proteins. Trends Genet 34:972-990. 10.1016/j.tig.2018.09.006

Gross I, Duluc I, Benameur T, Calon A, Martin E, Brabletz T, Kedinger M, Domon-Dell C, and Freund JN. 2008. The intestine-specific homeobox gene $\mathrm{Cd} \times 2$ decreases mobility and antagonizes dissemination of colon cancer cells. Oncogene 27:107-115. 10.1038/sj.onc.1210601

Hanouneh IA, Alkhouri N, and Singal AG. 2019. Hepatocellular carcinoma surveillance in the 21st century: Saving lives or causing harm? Clin Mol Hepatol. 10.3350/cmh.2019.1001

Hou L, Zhang X, Jiao Y, Li Y, Zhao Y, Guan Y, and Liu Z. 2019. ATP binding cassette subfamily B member 9 (ABCB9) is a prognostic indicator of overall survival in ovarian cancer. Medicine (Baltimore) 98:e15698. 10.1097/MD.0000000000015698

Huang Y, Fang C, Shi JW, Wen Y, and Liu D. 2017. Identification of hMex-3A and its effect on human bladder cancer cell proliferation. Oncotarget 8:61215-61225. 10.18632/oncotarget.18050

Jiang H, Zhang X, Luo J, Dong C, Xue J, Wei W, Chen J, Zhou J, Gao Y, and Yang C. 2012. Knockdown of hMex-3A by small RNA interference suppresses cell proliferation and migration in human gastric cancer cells. Mol Med Rep 6:575-580. 10.3892/mmr.2012.943

Jiao Y, Fu Z, Li Y, Meng L, and Liu Y. 2018. High EIF2B5 mRNA expression and its prognostic significance in liver cancer: a study based on the TCGA and GEO database. Cancer Manag Res 10:6003-6014. 10.2147/cmar.S185459

Jiao Y, Fu Z, Li Y, Zhang W, and Liu Y. 2019a. Aberrant FAM64A mRNA expression is an independent predictor of poor survival in pancreatic cancer. PLoS One 14:e0211291. 10.1371/journal.pone.0211291

Jiao Y, Li Y, Jiang P, Han W, and Liu Y. 2019b. PGM5: a novel diagnostic and prognostic biomarker for liver cancer. PeerJ 7:e7070. 10.7717/peerj.7070

Jiao Y, Li Y, Liu S, Chen Q, and Liu Y. 2019c. ITGA3 serves as a diagnostic and prognostic biomarker for pancreatic cancer. Onco Targets Ther 12:4141-4152. 10.2147/ott.S201675

Jiao Y, Li Y, Lu Z, and Liu Y. 2019d. High Trophinin-Associated Protein Expression Is an Independent Predictor of Poor Survival in Liver Cancer. Dig Dis Sci 64:137-143. 10.1007/s10620-018-5315-x

Peer] reviewing PDF | (2019:08:40097:1:0:NEW 24 Oct 2019) 
Kim MY, Hur J, and Jeong S. 2009. Emerging roles of RNA and RNA-binding protein network in cancer cells. BMB Rep 42:125-130.

Krepischi ACV, Maschietto M, Ferreira EN, Silva AG, Costa SS, da Cunha IW, Barros BDF, Grundy PE, Rosenberg C, and Carraro DM. 2016. Genomic imbalances pinpoint potential oncogenes and tumor suppressors in Wilms tumors. Mol Cytogenet 9:20. 10.1186/s13039-016-0227-y

Li Y, Jiao Y, Fu Z, Luo Z, SU J, and Li Y. 2019. High miR-454-3p expression predicts poor prognosis in hepatocellular carcinoma. Cancer Manag Res 11:2795-2802. 10.2147/CMAR.S196655

Masuda K, and Kuwano Y. 2019. Diverse roles of RNA-binding proteins in cancer traits and their implications in gastrointestinal cancers. Wiley Interdiscip Rev RNA 10:e1520. 10.1002/wrna.1520

Moore MJ. 2005. From birth to death: the complex lives of eukaryotic mRNAs. Science 309:1514-1518. 10.1126/science. 1111443

Pereira B, Le Borgne M, Chartier NT, Billaud M, and Almeida R. 2013a. MEX-3 proteins: recent insights on novel posttranscriptional regulators. Trends Biochem Sci 38:477-479. 10.1016/j.tibs.2013.08.004

Pereira B, Sousa S, Barros R, Carreto L, Oliveira P, Oliveira C, Chartier NT, Plateroti M, Rouault JP, Freund JN, Billaud $\mathrm{M}$, and Almeida R. 2013b. CDX2 regulation by the RNA-binding protein MEX3A: impact on intestinal differentiation and stemness. Nucleic Acids Res 41:3986-3999. 10.1093/nar/gkt087

Robin X, Turck N, Hainard A, Tiberti N, Lisacek F, Sanchez JC, and Muller M. 2011. pROC: an open-source package for $\mathrm{R}$ and $\mathrm{S}+$ to analyze and compare ROC curves. BMC Bioinformatics 12:77. 10.1186/1471-2105-12-77

Sheth U, and Parker R. 2003. Decapping and decay of messenger RNA occur in cytoplasmic processing bodies. Science 300:805-808. 10.1126/science.1082320

Shi JW, and Huang Y. 2017. Mex3a expression and survival analysis of bladder urothelial carcinoma. Oncotarget 8:54764-54774. 10.18632/oncotarget.18399

Sun Z, Sun L, He M, Pang Y, Yang Z, and Wang J. 2019. Low BCL7A expression predicts poor prognosis in ovarian cancer. J Ovarian Res 12:41. 10.1186/s13048-019-0518-0

Team RDCJC. 2009. R : A language and environment for statistical computing. R Foundation for Statistical Computing, Vienna, Austria. 14:12-21.

Therneau TM, and April. 1994. A Package for Survival Analysis in S.

Therneau TM, and Grambsch PM. 2000. Modeling Survival Data: Extending the Cox Model. New York: Springer.

Wong CM, Tsang FH, and Ng IO. 2018. Non-coding RNAs in hepatocellular carcinoma: molecular functions and pathological implications. Nat Rev Gastroenterol Hepatol 15:137-151. 10.1038/nrgastro.2017.169

Zhang C, Yang M, and Ericsson AC. 2019. Antimicrobial Peptides: Potential Application in Liver Cancer. Front Microbiol 10:1257. 10.3389/fmicb.2019.01257

\section{Appendices}

Figure legends:

Figure 1. Assessment of the relationship between MEX3A mRNA expression and clinical characteristics (A-I). Comparison of MEX3A mRNA expression in 373 cases of liver cancer and 50 normal liver tissues (A). Comparison of MEX3A mRNA expression according to clinical parameters: clinical stage (I, II, III and IV) (B), histologic grade (G1, G2, G3 and G4) (C), T classification (T1, T2, T3 and T4) (D), N classification (N0, N1 and NX) (E), M classification $(\mathrm{M} 0, \mathrm{M} 1$ and $\mathrm{MX})(\mathrm{F})$, age $(<55$ and $\geq 55)(\mathrm{G})$, gender (male and female) $(\mathrm{H})$ and vital status (I). 
321 Figure 2. ROC analysis of the sensitivity and specificity of MEX3A to assess liver cancer. ROC

322

323

324

325

326

327

328

329

330

331

332

333

334

335

336

337

338

339

340

341

342

343

344

345

346

347

348 curve for MEX3A expression in normal liver tissue and tumor tissue: $A U C=0.837$ (A). I stage patients: $\mathrm{AUC}=0.823$ (B). II stage patients: $\mathrm{AUC}=0.844$ (C). III stage patients: $\mathrm{AUC}=0.835$ (D). IV stage patients: $\mathrm{AUC}=0.888(\mathrm{E})$. (Abbreviations: AUC, area under the curve; ROC, receiveroperating characteristics).

Figure 3. Analysis of OS between high and low expression groups of MEX3A according to the different clinical variables of liver cancer patients. Kaplan-Meier curves of OS in all patients with liver cancer (A). Subgroup analysis was performed in stage I/II (B), stage III/IV (C), histological grade G1/G2 (D), histological grade G3/G4 (E), males (F), females (G), young patients (H) and old patients (I).

Figure 4. Analysis of RFS in high and low MEX3A expression groups according to the clinical variables of liver cancer patients. Kaplan-Meier curves of RFS in all patients with liver cancer (A). Subgroup analysis was performed in stage I/II (B), stage III/IV (C), histological grade G1/G2 (D), histological grade $\mathrm{G} 3 / \mathrm{G} 4(\mathrm{E})$, males $(\mathrm{F})$, females $(\mathrm{G})$, young patients $(\mathrm{H})$ and old patients $(\mathrm{I})$.

Figure S1. Optimal cutoff points for dividing patients into high and low MEX3A expression groups identified through ROC curves.

\section{Table Legends:}

Table 1. MEX3A mRNA expression and clinical characteristics in liver cancer patients.

Table 2. Association between MEX3A expression and clinical characteristics in liver cancer patients. (\% represents the distribution of different clinical features in the single MEX3A expression group)

Table 3. Relationship between clinical parameters, MEX3A mRNA expression and overall survival in liver cancer patients.

Table 4. Relationship between clinical parameters, MEX3A mRNA expression and relapse-free survival in liver cancer patients. 


\section{Table 1 (on next page)}

Table 1. MEX3A mRNA expression and clinical characteristics in liver cancer patients.

NA: not available 
1 Table 1. MEX3A mRNA expression and clinical characteristics in liver cancer patients.

\begin{tabular}{lc}
\hline characteristics & Number of pts(\%) \\
\hline age & \\
$<55$ & $117(31.45)$ \\
$>=55$ & $255(68.55)$ \\
NA & $1(0.00)$ \\
gender & \\
FEMALE & $121(32.44)$ \\
MALE & $252(67.56)$ \\
histological_type & \\
Fibrolamellar_Carcinoma & $3(0.8)$ \\
Hepatocellular Carcinoma & $363(97.32)$ \\
Hepatocholangiocarcinoma & $7(1.88)$ \\
(Mixed) & \\
histologic_grade & \\
G1 & $55(14.75)$ \\
G2 & $178(47.72)$ \\
G3 & $123(32.98)$ \\
G4 & $12(3.22)$ \\
NA & $5(1.34)$ \\
stage & \\
I & $172(46.11)$ \\
II & $87(23.32)$ \\
III & $85(22.79)$ \\
IV & $5(1.34)$ \\
NA & $24(6.43)$ \\
T_classification & \\
T1 & $182(48.79)$ \\
T2 & $95(25.47)$
\end{tabular}




$\begin{array}{cc}\text { T3 } & 80(21.45) \\ \text { T4 } & 13(3.49) \\ \text { TX } & 1(0.27) \\ \text { NA } & 2(0.54) \\ \text { N_classification } & \\ \text { N0 } & 253(67.83) \\ \text { N1 } & 4(1.07) \\ \text { NX } & 115(30.83) \\ \text { NA } & 1(0.27) \\ \text { M_classification } & \\ \text { M0 } & 267(71.58) \\ \text { M1 } & 4(1.07) \\ \text { MX } & 102(27.35) \\ \text { radiation_therapy } & \\ \text { NO } & 340(91.15) \\ \text { YES } & 8(2.14) \\ \text { NA } & 25(6.7) \\ \text { residual_tumor } & \\ \text { R0 } & 326(87.4) \\ \text { R1 } & 17(4.56) \\ \text { R2 } & 1(0.27) \\ \text { RX } & 22(5.9) \\ \text { NA } & 7(1.88) \\ \text { vital_status } & \\ \text { DECEASED } & 130(34.85) \\ \text { LIVING } & 243(65.15) \\ \text { relapse } & \\ \text { NO } & 179(55.94) \\ \text { YES } & 141(44.06) \\ & \end{array}$


NA

MEX3A

high

low

NA: not available
$53(14.2)$

117(31.37)

256(68.63) 


\section{Table 2 (on next page)}

Table 2. Association between MEX3A expression and clinical characteristics in liver cancer patients.

$\%$ represents the distribution of different clinical features in the single MEX3A expression group $P$-value in bold represent significant clinical significance $(p<$ 0.05) 
Table 2. Association between MEX3A expression and clinical characteristics in liver cancer patients.

\begin{tabular}{|c|c|c|c|c|c|c|c|c|}
\hline \multirow{2}{*}{ Clinical characteristics } & \multirow[b]{2}{*}{ Variable } & \multirow[b]{2}{*}{ No. of patients } & \multicolumn{4}{|c|}{ MEX3A expression } & \multirow[b]{2}{*}{$\chi^{2}$} & \multirow[b]{2}{*}{ p-value } \\
\hline & & & High & $\%$ & Low & $\%$ & & \\
\hline \multirow[t]{2}{*}{ age } & $<55$ & 117 & 51 & $(43.59)$ & 66 & $(25.88)$ & 10.8573 & 0.001 \\
\hline & $>=55$ & 255 & 66 & $(56.41)$ & 189 & $(74.12)$ & & \\
\hline \multirow[t]{2}{*}{ gender } & FEMALE & 121 & 43 & $(36.75)$ & 78 & $(30.47)$ & 1.1741 & 0.279 \\
\hline & MALE & 252 & 74 & $(63.25)$ & 178 & $(69.53)$ & & \\
\hline \multirow[t]{3}{*}{ histological type } & Fibrolamellar Carcinoma & 3 & 0 & $(0)$ & 3 & $(1.17)$ & 1.8006 & 0.547 \\
\hline & Hepatocellular Carcinoma & 363 & 114 & $(97.44)$ & 249 & $(97.27)$ & & \\
\hline & Hepatocholangiocarcinoma (Mixed) & 7 & 3 & $(2.56)$ & 4 & $(1.56)$ & & \\
\hline \multirow[t]{4}{*}{ histologic grade } & G1 & 55 & 10 & $(8.7)$ & 45 & $(17.79)$ & 20.0434 & 0.000 \\
\hline & $\mathrm{G} 2$ & 178 & 48 & $(41.74)$ & 130 & $(51.38)$ & & \\
\hline & G3 & 123 & 48 & $(41.74)$ & 75 & $(29.64)$ & & \\
\hline & G4 & 12 & 9 & $(7.83)$ & 3 & $(1.19)$ & & \\
\hline \multirow[t]{4}{*}{ stage } & I & 172 & 48 & $(43.64)$ & 124 & $(51.88)$ & 2.1216 & 0.512 \\
\hline & II & 87 & 30 & $(27.27)$ & 57 & $(23.85)$ & & \\
\hline & III & 85 & 30 & $(27.27)$ & 55 & $(23.01)$ & & \\
\hline & IV & 5 & 2 & $(1.82)$ & 3 & $(1.26)$ & & \\
\hline \multirow[t]{5}{*}{$\mathrm{T}$ classification } & $\mathrm{T} 1$ & 182 & 49 & $(41.88)$ & 133 & $(52.36)$ & 4.9613 & 0.253 \\
\hline & $\mathrm{T} 2$ & 95 & 35 & $(29.91)$ & 60 & $(23.62)$ & & \\
\hline & $\mathrm{T} 3$ & 80 & 27 & $(23.08)$ & 53 & $(20.87)$ & & \\
\hline & $\mathrm{T} 4$ & 13 & 6 & $(5.13)$ & 7 & $(2.76)$ & & \\
\hline & TX & 1 & 0 & $(0)$ & 1 & $(0.39)$ & & \\
\hline \multirow[t]{3}{*}{$\mathrm{N}$ classification } & No & 253 & 86 & $(73.5)$ & 167 & $(65.49)$ & 3.4688 & 0.135 \\
\hline & N1 & 4 & 2 & $(1.71)$ & 2 & $(0.78)$ & & \\
\hline & NX & 115 & 29 & $(24.79)$ & 86 & (33.73) & & \\
\hline \multirow[t]{3}{*}{ M classification } & M0 & 267 & 88 & $(75.21)$ & 179 & $(69.92)$ & 2.0953 & 0.304 \\
\hline & M1 & 4 & 2 & $(1.71)$ & 2 & $(0.78)$ & & \\
\hline & MX & 102 & 27 & $(23.08)$ & 75 & (29.3) & & \\
\hline
\end{tabular}




\begin{tabular}{|c|c|c|c|c|c|c|c|c|}
\hline \multirow[t]{2}{*}{ radiation therapy } & NO & 340 & 106 & $(98.15)$ & 234 & $(97.5)$ & 0 & 1.000 \\
\hline & YES & 8 & 2 & $(1.85)$ & 6 & $(2.5)$ & & \\
\hline \multirow[t]{4}{*}{ residual tumor } & R0 & 326 & 97 & $(84.35)$ & 229 & $(91.24)$ & 5.1533 & 0.134 \\
\hline & $\mathrm{R} 1$ & 17 & 7 & (6.09) & 10 & $(3.98)$ & & \\
\hline & $\mathrm{R} 2$ & 1 & 0 & $(0)$ & 1 & $(0.4)$ & & \\
\hline & $\mathrm{RX}$ & 22 & 11 & $(9.57)$ & 11 & $(4.38)$ & & \\
\hline \multirow[t]{2}{*}{ vital status } & DECEASED & 130 & 55 & $(47.01)$ & 75 & $(29.3)$ & 10.3281 & 0.001 \\
\hline & LIVING & 243 & 62 & $(52.99)$ & 181 & $(70.7)$ & & \\
\hline
\end{tabular}

$3 \quad P$-value in bold represent significant clinical significance $(p<0.05)$ 


\section{Table 3(on next page)}

Table 3. Relationship between clinical parameters, MEX3A mRNA expression and overall survival in liver cancer patients.

$P$-value in bold represent significant clinical significance $(p<0.05)$ 
1 Table 3. Relationship between clinical parameters, MEX3A mRNA expression and overall survival in liver cancer patients.

\begin{tabular}{|c|c|c|c|c|c|c|}
\hline \multirow[b]{2}{*}{ Parameters } & \multicolumn{3}{|c|}{ Univariate analysis } & \multicolumn{3}{|c|}{ Multivariate analysis } \\
\hline & Hazard Ratio & 95\%CI (lower upper) & Pvalue & $\sqsubset$ Hazard Ratio & 95\%CI (lower-upper) & Pvalue \\
\hline age & 1.00 & $0.69-1.45$ & 0.997 & & & \\
\hline gender & 0.80 & $0.56-1.14$ & 0.220 & & & \\
\hline histological type & 0.99 & $0.27-3.66$ & 0.986 & & & \\
\hline histologic grade & 1.04 & $0.84-1.3$ & 0.698 & & & \\
\hline stage & 1.38 & $1.15-1.66$ & 0.001 & 0.86 & $0.69-1.07$ & 0.163 \\
\hline $\mathrm{T}$ classification & 1.66 & $1.39-1.99$ & 0.000 & 1.88 & $1.48-2.38$ & 0.000 \\
\hline $\mathrm{N}$ classification & 0.73 & $0.51-1.05$ & 0.086 & & & \\
\hline M classification & 0.72 & $0.49-1.04$ & 0.077 & & & \\
\hline radiation therapy & 0.51 & $0.26-1.03$ & 0.060 & & & \\
\hline residual tumor & 1.42 & $1.13-1.8$ & 0.003 & 1.33 & $1.03-1.71$ & 0.026 \\
\hline MEX3A & 2.29 & $1.61-3.26$ & 0.000 & 2.26 & $1.58-3.23$ & 0.000 \\
\hline
\end{tabular}

$P$-value in bold represent significant clinical significance $(p<0.05)$ 


\section{Table 4(on next page)}

Table 4. Relationship between clinical parameters, MEX3A mRNA expression and relapse-free survival in liver cancer patients.

$P$-value in bold represent significant clinical significance $(p<0.05)$ 
Table 4. Relationship between clinical parameters, MEX3A mRNA expression and relapse-free survival in liver cancer patients.

\begin{tabular}{lccccccc}
\hline$\square$ & \multicolumn{3}{c}{ Univariate analysis } & & \multicolumn{3}{c}{ Multivariate analysis } \\
\cline { 2 - 4 } Parameters & Hazard Ratio & $95 \%$ CI (lower upper) & Pvalue & & Hazard Ratio & 95\%CI (lower-upper) & Pvalue \\
\hline age & 0.90 & $0.63-1.28$ & 0.550 & & & \\
gender & 0.99 & $0.7-1.41$ & 0.966 & & & \\
histological type & 2.02 & $0.66-6.24$ & 0.220 & & & \\
histologic grade & 0.98 & $0.8-1.21$ & 0.883 & & & \\
stage & 1.66 & $1.38-1.99$ & 0.000 & 1.13 & $0.87-1.46$ & 0.358 \\
T classification & 1.78 & $1.49-2.12$ & 0.000 & & 1.69 & $1.29-2.21$ & $\mathbf{0 . 0 0 0}$ \\
N classification & 0.97 & $0.67-1.4$ & 0.874 & & & \\
M classification & 1.17 & $0.79-1.74$ & 0.432 & & & \\
radiation therapy & 0.74 & $0.26-2.16$ & 0.584 & & & \\
residual tumor & 1.28 & $1.01-1.61$ & 0.042 & 1.32 & $1.04-1.67$ & $\mathbf{0 . 0 2 4}$ \\
MEX3A & 2.05 & $1.46-2.9$ & 0.000 & 2.19 & $\mathbf{0 . 0 0 0}$ \\
\hline
\end{tabular}




\section{Figure 1}

Figure 1. Assessment of the relationship between MEX3A mRNA expression and clinical characteristics (A-I).

Comparison of MEX3A mRNA expression in 373 cases of liver cancer and 50 normal liver tissues (A). Comparison of MEX3A mRNA expression according to clinical parameters: clinical stage (I, II, III and IV) (B), histologic grade (G1, G2, G3 and G4) (C), T classification (T1, T2, T3 and T4) (D), N classification (N0, N1 and NX) (E), M classification (M0, M1 and MX) (F), age $(<55$ and $\geq 55)(G)$, gender (male and female) $(H)$ and vital status (I). 
A

MEX3A expression in tumor vs normal

$$
\text { type 追 tumor 追 nommat }
$$

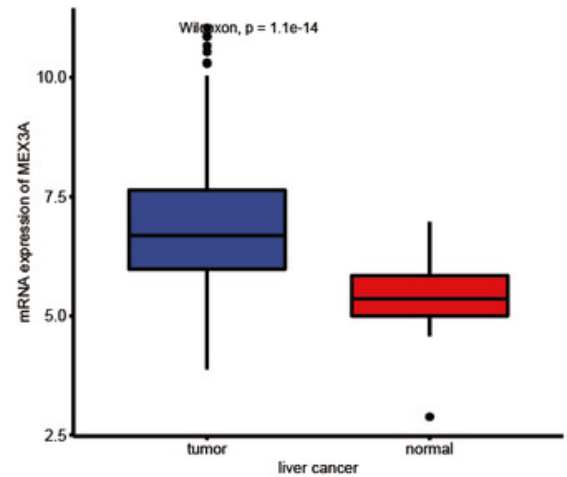

D

MEX3A expression grouped by $T$ classification

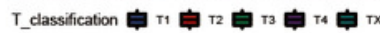

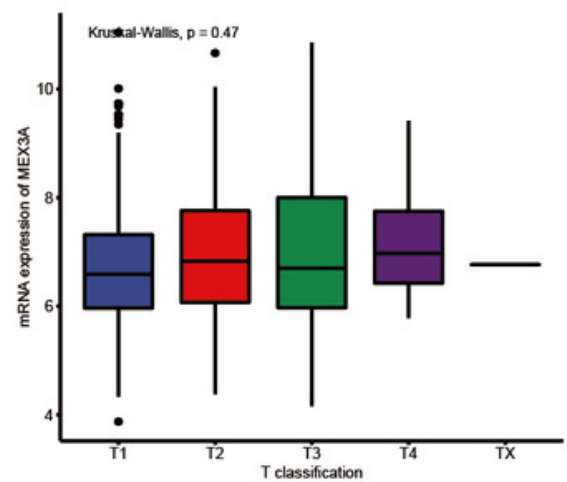

G

MEX3A expression grouped by age

$$
\text { age 追 } 455 \text { 官 > } 55
$$

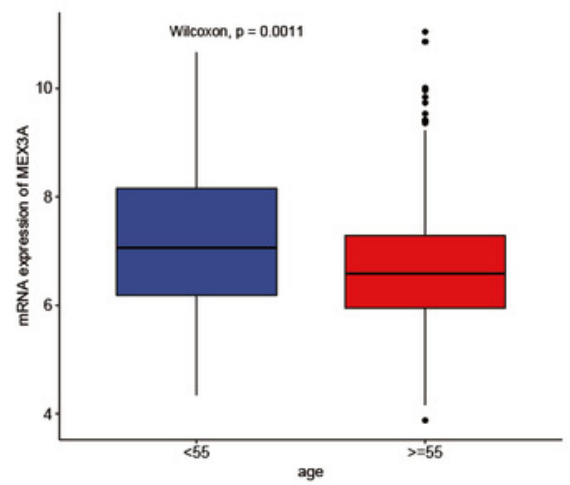

B

MEX3A expression grouped by stage

stage 追 |白" 追

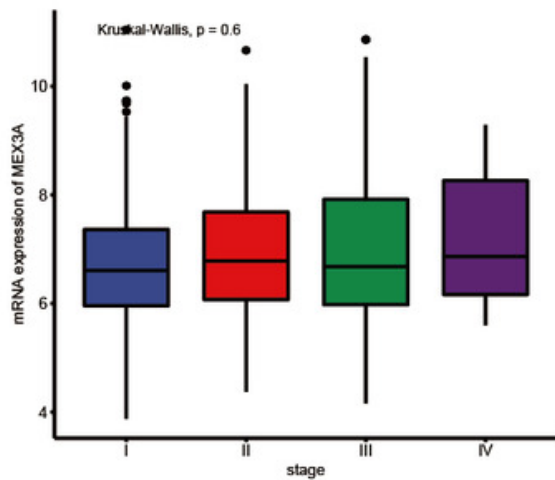

E

MEX3A expression grouped by $\mathrm{N}$ classification N_classification 追 ${ }^{N}$ 追 ${ }^{N}$ 白 $N x$

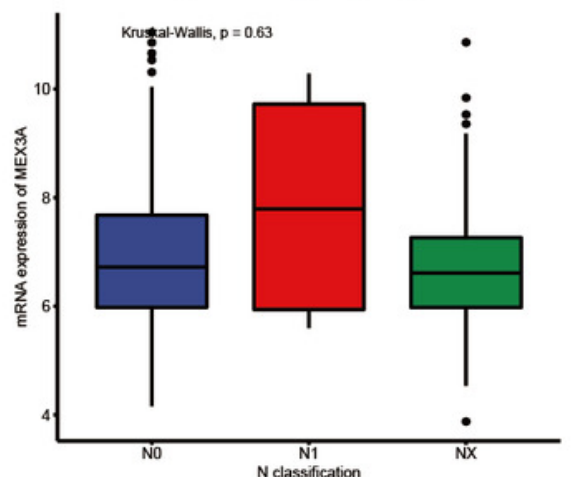

$\mathrm{H}$

MEX3A expression grouped by gender gender 官 MaLE 早 FEMaLE

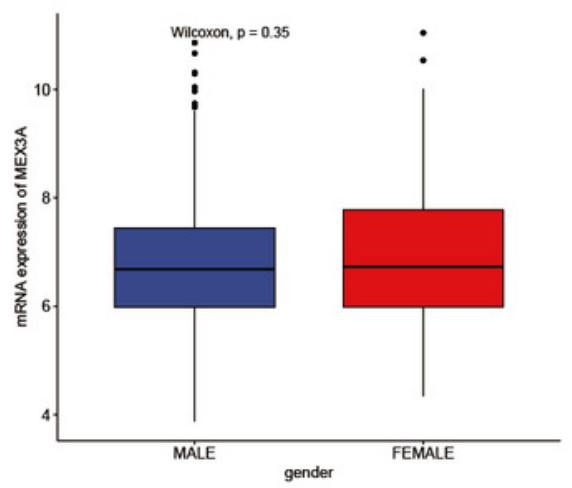

C

MEX3A expression grouped by histologic grade

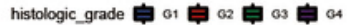

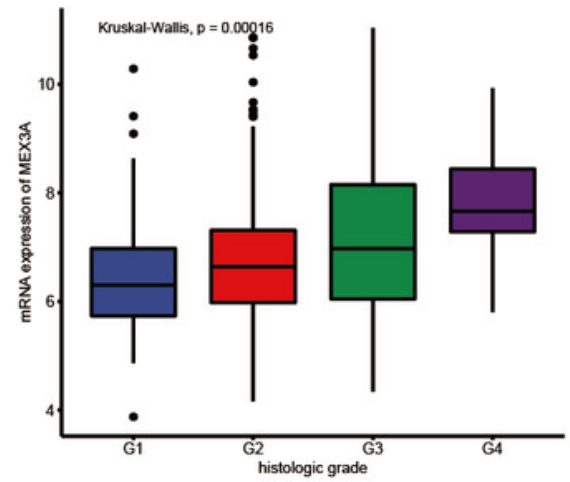

F

MEX3A expression grouped by $M$ classification

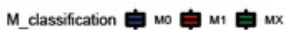

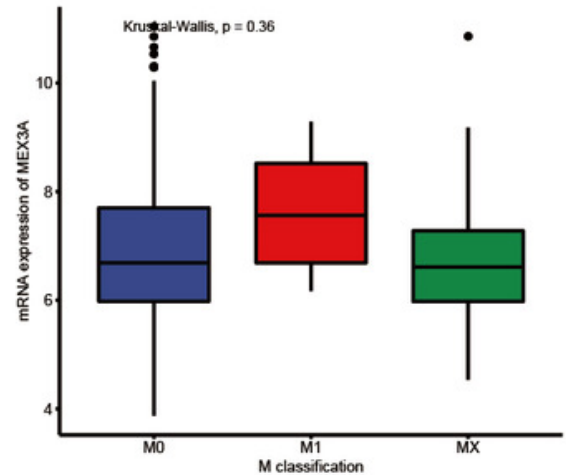

I

MEX3A expression grouped by vital status vital_status 追 LNNwo 追 DECEAsED

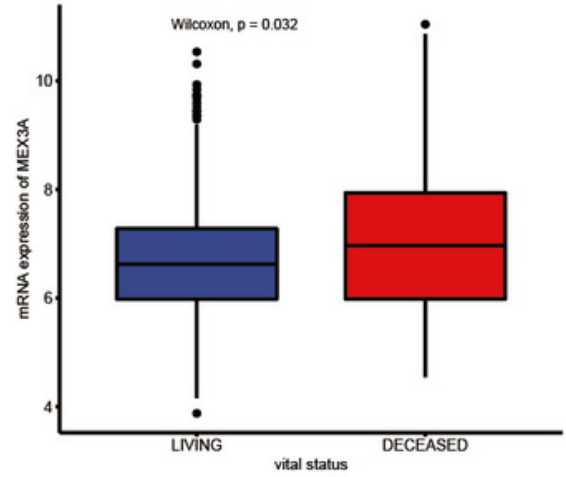




\section{Figure 2}

ROC analysis of the sensitivity and specificity of MEX3A to assess liver cancer.

ROC curve for MEX3A expression in normal liver tissue and tumor tissue: $A U C=0.837(A)$. I stage patients: $A U C=0.823$ (B). II stage patients: $A U C=0.844$ (C). III stage patients: $A U C=0.835(D)$. IV stage patients: $A U C=0.888(E)$. (Abbreviations: $A U C$, area under the curve; ROC, receiver-operating characteristics).

A

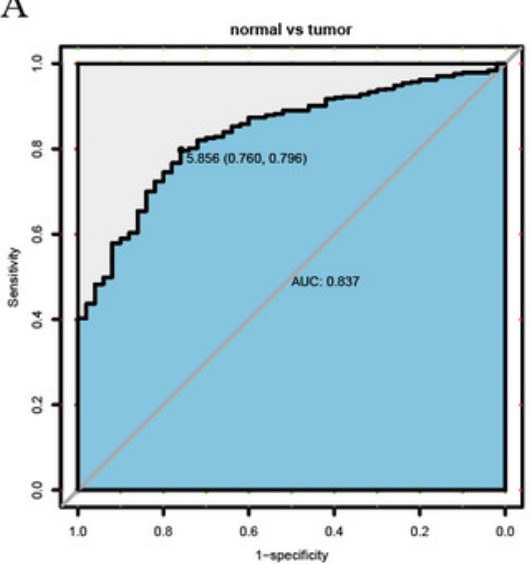

$\mathrm{D}$

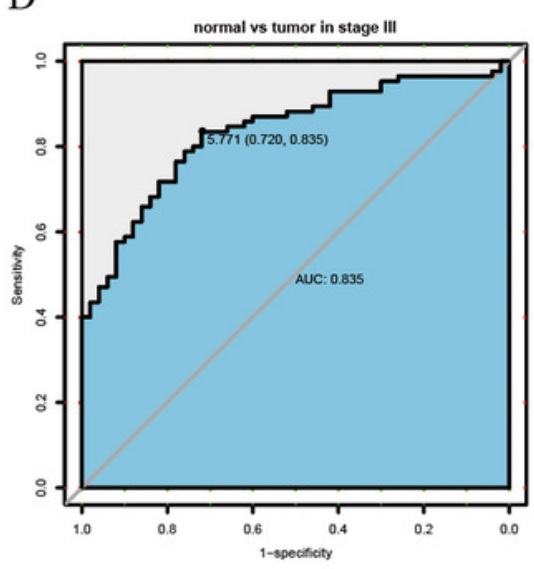

B

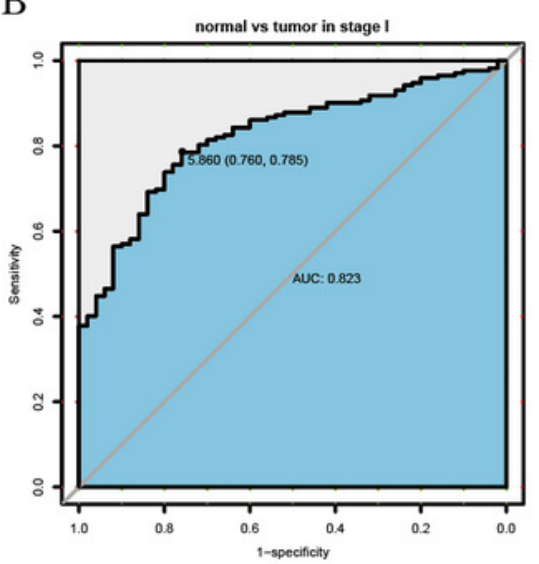

E

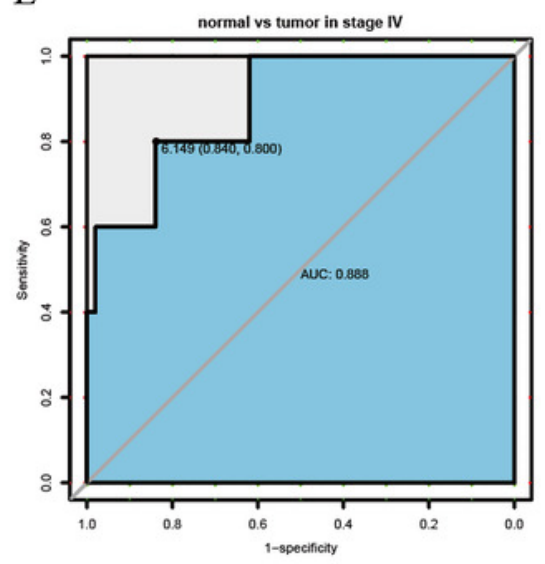

C

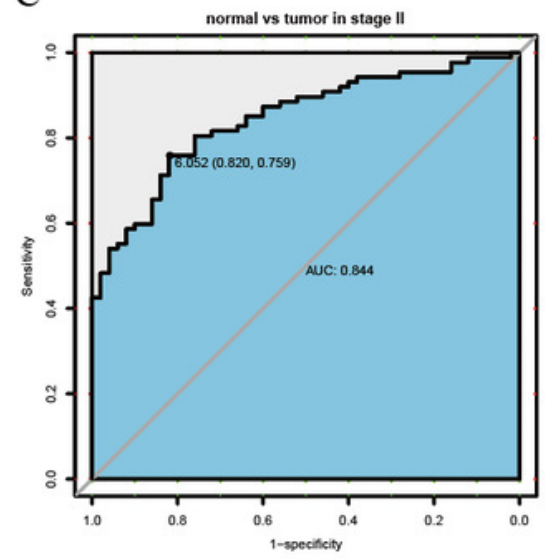




\section{Figure 3}

Figure 3. Analysis of OS between high and low expression groups of MEX3A according to the different clinical variables of liver cancer patients.

Kaplan-Meier curves of OS in all patients with liver cancer (A). Subgroup analysis was performed in stage I/II (B), stage III/IV (C), histological grade G1/G2 (D), histological grade G3/G4 (E), males (F), females (G), young patients $(\mathrm{H})$ and old patients (I). 
A

Overall survival group by MEX3A in all tumors Strata $=$ MEcasanion + MExiantow

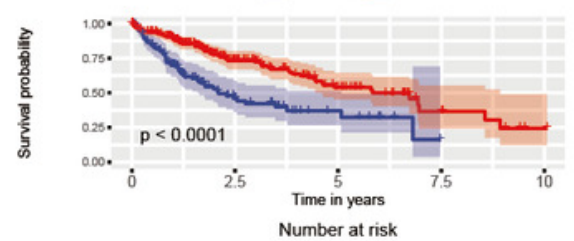

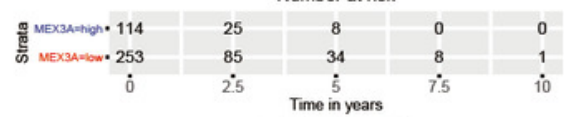

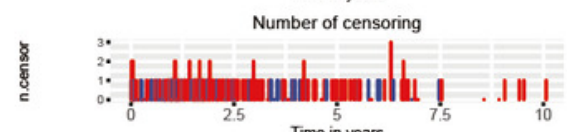

D

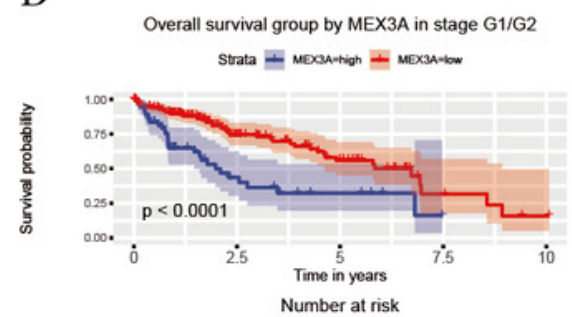

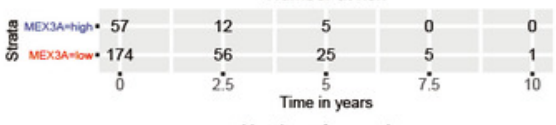

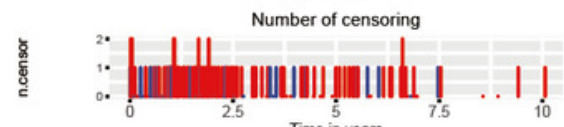

G

Overall survival group by MEX3A in FEMALE Strata II MexaAnion II MEx3A=6ow

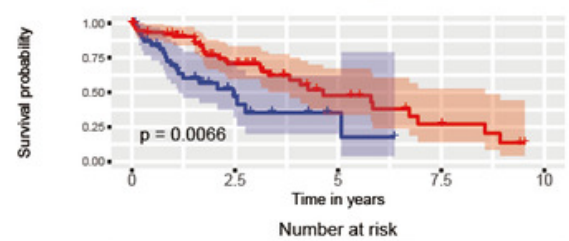

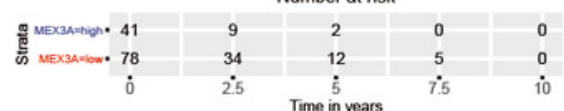

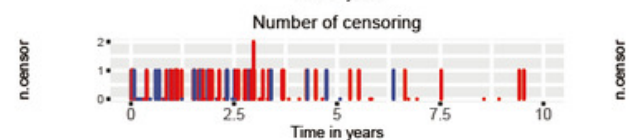
Time in years
B

Overall survival group by MEX3A in stage I/I Strata $I$ Mexantion $I$ Mexantious

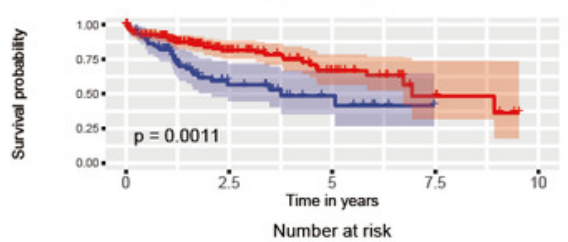

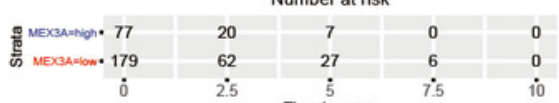

Time in years

3.
2.
0.

E

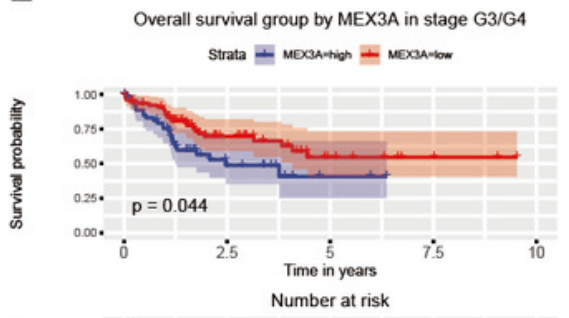

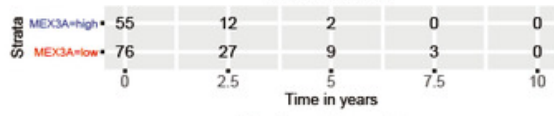

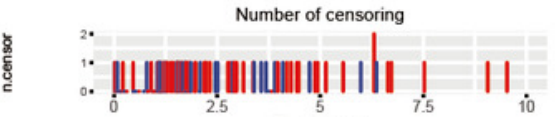

$\mathrm{H}$

Overall survival group by MEX3A in younger

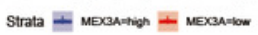

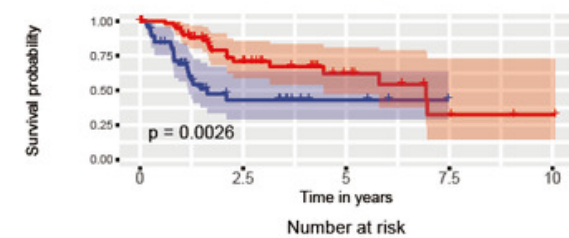

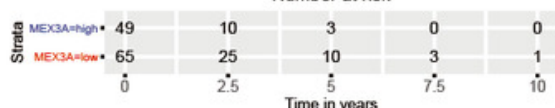

Number of censoring

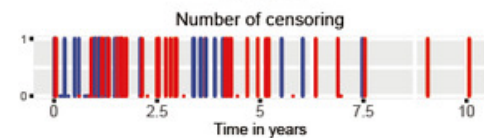

C

Overall survival group by MEX3A in stage III/IV

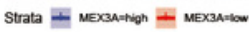

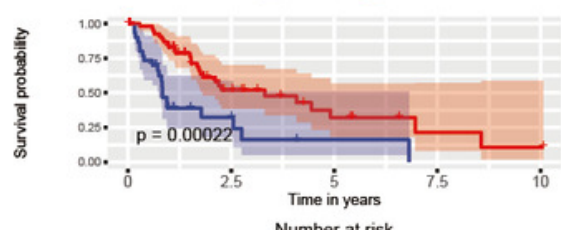

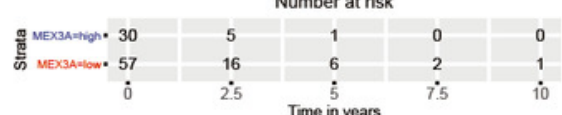
Number of censoring

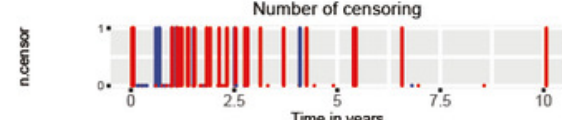

F

F Overall survival group by MEX 3 A in MALE Strata II MexaA-ison - MexaA-low

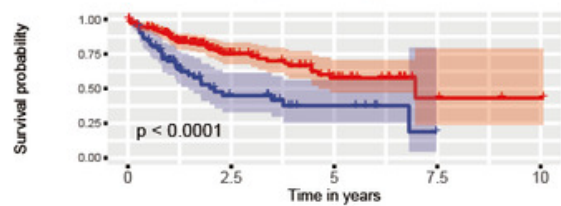
Number at risk

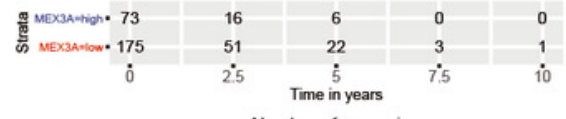

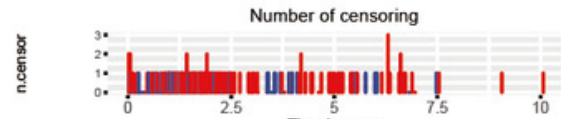

I

Trme in years

Overall survival group by MEX $3 A$ in older

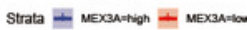

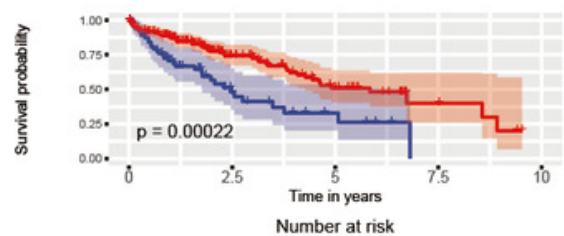

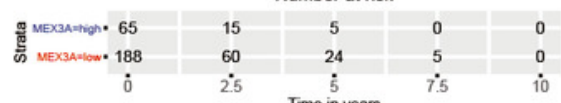

c. 


\section{Figure 4}

Figure 4. Analysis of RFS in high and low MEX3A expression groups according to the clinical variables of liver cancer patients.

Kaplan-Meier curves of RFS in all patients with liver cancer (A). Subgroup analysis was performed in stage I/II (B), stage III/IV (C), histological grade G1/G2 (D), histological grade G3/G4 (E), males (F), females (G), young patients $(\mathrm{H})$ and old patients (I). 
A

Relapse free survival group by MEX3A in all tumors

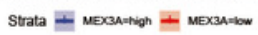

衰

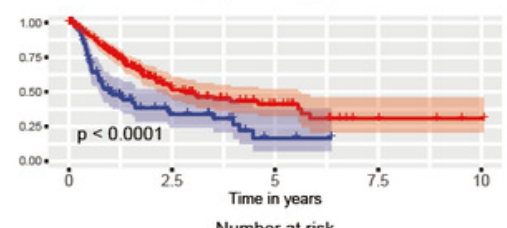

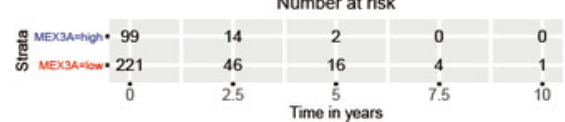

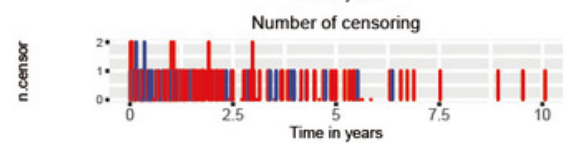

D

Relapse free survival group by MEX $3 A$ in stage G1/G2

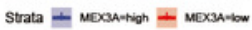

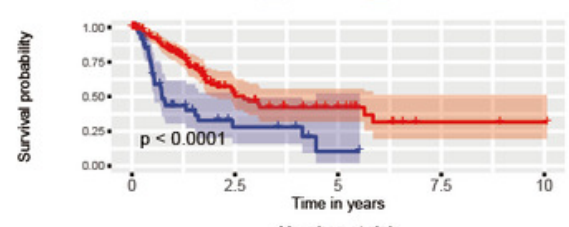

Number at risk

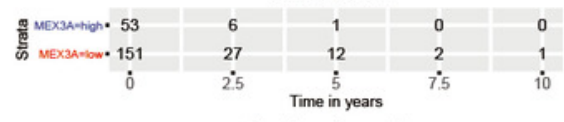

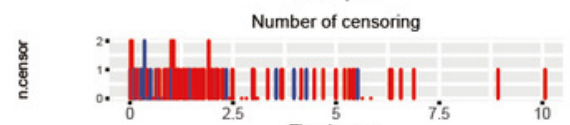

G

Relapse free survival group by MEX3A in FEMALE

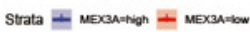

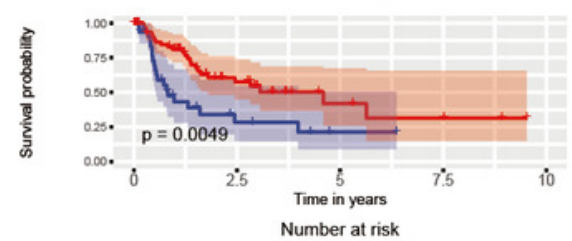

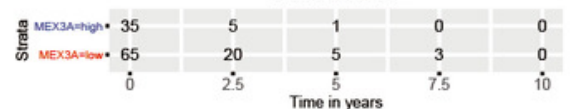

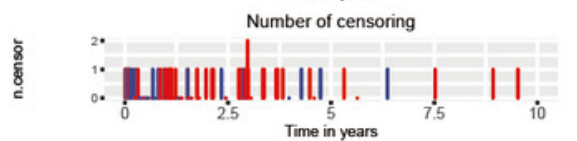

B

Relapse free survival group by MEX $3 \mathrm{~A}$ in stage $1 / 11$

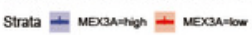

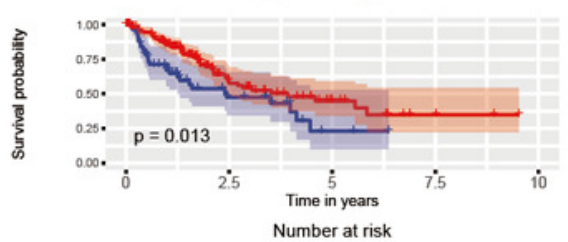

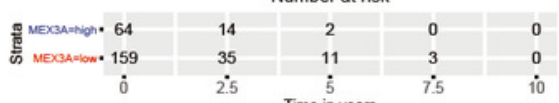

Number of censoring

ङू

E

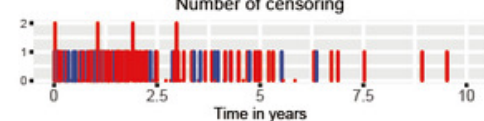

Time in year

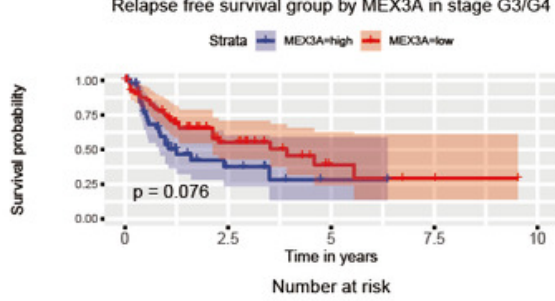

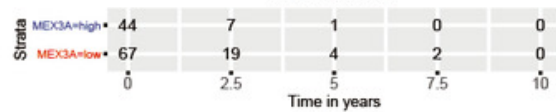

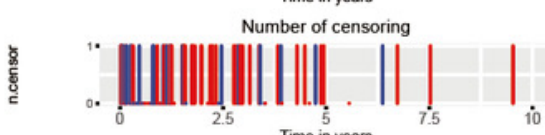

$\mathrm{H}$

Relapse free survival group by MEX3A in younger

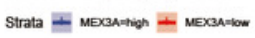

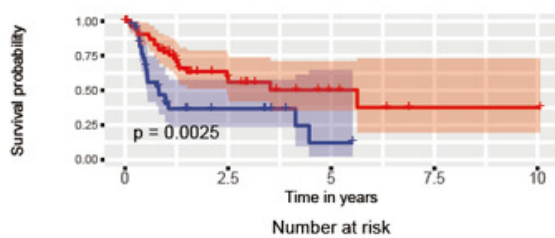

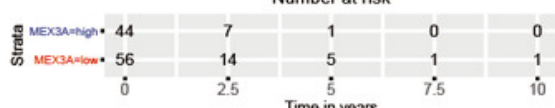

Number of censoring

2.
C

Relapse free survival group by MEX $3 \mathrm{~A}$ in stage III/IV Strata + MexaAnion + Mex3A-low

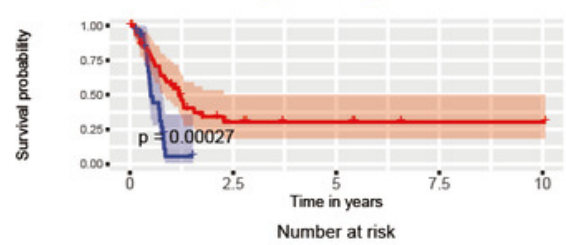

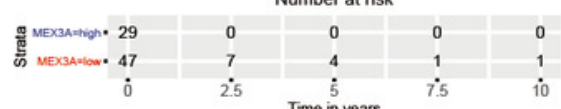

Number of censoring

离

$\mathrm{F}$

Relapse free survival group by MEX3A in MALE

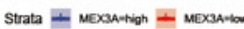

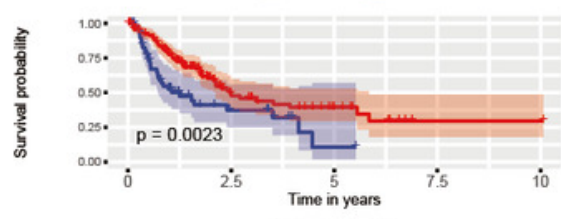

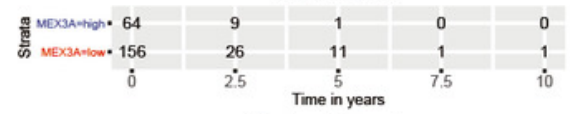

Number of censoring

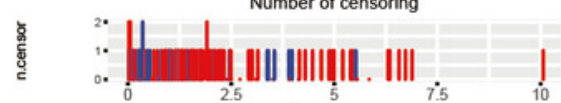

I

Relapse free survival group by MEX3A in older

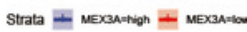

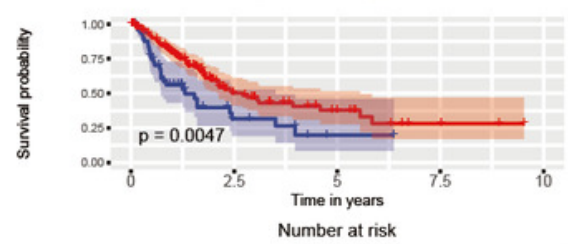

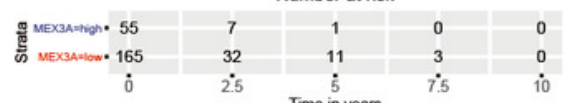
Number of censoring 